\title{
EVALUATION OF POST OPERATIVE PERITONEAL LAVAGE TO PREVENT RECURRENT ADHESIVE INTESTINAL OBSTRUCTION
}

\author{
Mostafa Mohammed El Saady* MD, Khaled Abd-El Azeez Metwally* MD, Mohamed Rashad Hefny* \\ MD, Waleed Ahmed Abd-El Hady*M.Sc. \\ General Surgery department, Faculty of Medicine, Zagazig University
}

\section{ABSTRACT}

Objective: to evaluate the effectiveness of postoperative peritoneal lavage (PPL) in prevention of recurrent Adhesive Small Bowel Obstruction (ASBO).Patients and methods: a total of 36 patients were included in this study. All of these patients suffered from ASBO. They were randomized into two groups. Group I (18 patients) were treated with adhesiolysis followed by PPL and group II (18 patients) were treated with adhesiolysis alone. Patients were followed up for an average period of 15 months to detect the incidence of recurrent ASBO. Results: :In group1 only one (5.6\%) patient had an attack of ASBO which responded to conservative management and did not need redo operation while in group II seven $(38.9 \%)$ patients developed a recurrent episode of ASBO four of them(22.2\%)needed surgical re exploration. So the rate of surgical reoperation in group 1 was zero in comparison to group 2 which was( 16.6\%). Conclusion: PPL is a new hope for prevention of recurrent ASBO that achieved statistically significant reduction in the recurrence rate and surgical redo.
\end{abstract}

\section{INTRODUCTION}

$\mathrm{T}$ he incidence of intra-abdominal adhesions ranges from $67 \%$ to $93 \%$ after general surgical abdominal operations and from $60 \%$ to $90 \%$ after gynecological procedures. Not unexpectedly, adhesion formation is considered one of the most common post-operative complications (1).

They can induce a broad range of diseases such as infertility, pain, bowel obstruction, and difficulties experienced during re-operative interventions $(\mathbf{2 , 3 , 4 )}$.

Peritoneal adhesions generally form in the early postoperative period. For decades, unremitting efforts on the issue are focused on developing products used during laparotomy. Numerous drugs against postoperative adhesion have been tested, and have shown promise in animal models, but few have penetrated into clinical practice $(\mathbf{5 , 6})$.

Postoperative adhesions represent a significant socioeconomic burden on health care resources. For example in 1994, the estimated financial impact for direct patient care owing to adhesions related disorders in the United States of America was 1.3 billion US\$, while in Sweden, it is estimated that health care burden owing to adhesive disease reaches 13 million US\$ annually

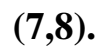

Understanding of the patho-physiology of adhesion formation is of outmost importance to discover an effective method to reduce or abolish adhesion formation as well as identification of inflammatory mechanisms: cutting, abrasion, ischaemia, desiccation and coagulation $(\mathbf{9 , 1 0})$. The fluid exudates released from injured peritoneal surfaces is rich in plasma protein especially fibrinogen. Activation of the coagulation cascade results in formation of fibrin. Fibrin is tacky substance and causes injured serosal surfaces to coalesce $(\mathbf{1 1 , 1 2})$.

As laparotomies are more frequent performed in surgical practice the number of small bowel obstruction secondary to post operative adhesion tend to increase steadly. The preventive measures against adhesion formation are of considerable clinical importance. Surgical separation for adhesions prophylaxis can potentially be achieved by using solid or mechanical barriers or by intraperitoneal solutions (13).

Fibrinolysis allows mesothelial cells to proliferate and peritoneal defect to be restored within 4 to 5 days preventing permanent attachment of adjacent surface. If fibrinolysis doesnot occure within 5 to 7 days of peritoneal injury, fibrin matrix persists and gradually becomes more organized as collagen secreting fibroblasts and reparative cells infiltrate the matrix.. (11).

The goal of adhesion prevention is to abolish or reduce the incidence, severity, extent and consequences of adhesions while retaining normal healing and preventing infection. Over the years, several strategies to prevent postoperative adhesion formation have been proposed, based on what has been learned about the underlying pathophysiology (6).

Postoperative Peritoneal Lavage (PPL) is an idea developed to minimize postoperative adhesions based on the observation that patients who developedpostoperative ascites rarely complain of adhesions related disorders

PATIENTS AND METHODS

This study included 36 patients with postoperative adhesive intestinal obstruction 
who were admitted to the emergency unit of our department of General Surgery Zagazig University hospitals from October 2012 to July 2014

Inclusion criteria:

Patients admitted to the hospital with provisional diagnosis of first attack of ASBO. ASBO is diagnosed clinically by the tetrad of colicky pain, vomiting, absolute constipation and abdominal distension and radiologically by multiple fluid levels in plain erect of the abdomen together with history of previous laparotomy

Exclusion criteria:

1. Patients under 18 years and above 60 years

2. Patients responded to ,conservative measures and did not need surgical exploration

3. Patients who have ascites at presentation.

4. Patients who had a previous episode of ASBO.

5. Patients whose explorations revealed gangrenous intestinal loop that necessitated resection anastomosis or septic lesion that needed rubber drains insertion

6. Patients with cardiac or renal insufficiency for fear of lavage induced hypervolaemia

7. Patients whose explorations revealed intestinal obstruction due to any cause other than adhesions.

8. Patient who died during the follow up period

9. Patient who were missed during the follow up period.

All patients were admitted to the emergency unit and received conservative measures that included nasogastric tube insertion, intravenous fluids therapy, correction of blood electrolytes and repeated enemata

All patients included in the study failed to respond to conservative measures and were surgically explored for adhesiolysis. Each patient in the study was serially numbered and the patients were randomized into two equal groups:

Group I patients were treated with adhesiolysis plus PPL. After adhesiolysis, two
Nelaton catheters were inserted, the first in the right mid-axillary line just below the costal margin and placed above the liver. The second catheter is introduced in the left mid-axillary line above the iliac crest and was passed in the left paracolic gutter to settle in the pelvis.PPL is performed using one liter of warm (about $37.5^{\circ} \mathrm{c}$ ) saline infused through the upper catheter (the lavage tube) while the lower catheter (the drainage tube) is clamped and saline is retained in the peritoneal cavity for 8 hours till the next lavage where the drainage tube is declamped and left open while the patient in the semi-setting position till all of the intraperitoneal fluid is drained then the tube is clamped again and the next liter of saline is infused through the lavage tube. Then the process is repeated every 8 hours till return of intestinal motions but not less than three days, then the tubes are withdrawn. Intra-abdominal pressure is measured after each infusion. It is planned not to allow it to reach $10 \mathrm{~cm}$ water. It was noted that the amount of the drainage fluid was usually less than the infused one mostly due to absorption of saline through the peritoneum.

Group II patients for whom adhesiolysis was performed alone and the abdomen was closed without drains. All patients in group I were given explanation of the new technique and they signed a written consent form. Recurrent intestinal obstruction is diagnosed in the same parameters mentioned before

All- patients were followed up either through regular visits in the outpatient clinics at 6 months intervals.

\section{RESULTS}

In this study the male (21) to female (15) ratio for the patients with adhesive intestinal obstruction (1.4:1) Figure (1), and the age ranges in Group I from 19 to 60 years with mean of age was 34.2 years $(\mathbf{\pm 1 0 . 4})$ and in Group II from 20 to 60 years with mean of age was 33 years $( \pm \mathbf{1 1 . 5})$. The highest age incidence was found to be in the third decade of life (15 patients) as adhesive intestinal obstruction are more common in males than females, the patients aged below 18 years are excluded as they cannot give consent. The primary surgeries before ASBO are illustrated in, table 1.

In Group I, It was found that 17 patients (94.4\%) had no recurrent attaks of adhesive intestinal obstruction and 1 patient $(5.6 \%)$ had had one attak of recurrent adhesive intestinal obstruction during the $1^{\text {st }} 6$ months of the follow 
up period and re admitted again and managed conservatively without surgical intervention and discharged after 3 days of admission. In Group II, It was found that 11 patients $(61.1 \%)$ had no recurrent attaks of adhesive intestinal obstruction and 7 patients $(38.9 \%)$ had recurrent attaks adhesive intestinal obstruction. table 2, Figure (2).Four(22.2\%) of these recurrent patients needed surgical re exploration while three(16.6\%) are managed conservatively.

There was statistically significant reduction $\quad(\mathrm{P}<0.05)$.in the recurrence rate and surgical redo in group $\mathrm{I}$ in comparison to group II (table 3, Figure 3)

Also, it is worth to mention that one patient (5.6 $\%$ ) of the group I developed incisional hernia at his laparotomy scar 6 months postoperatively and was readmitted to hospital for its repair. Exploration of the peritoneal cavity during hernia repair revealed no intestinal adhesions at all.

Complications recorded with the technique:-Leak of the lavage fluid around the catheter may be due to large stab for insertion of the tube.

Some patients experienced some abdominal discomfort at the end of the lavage, may be due to rapid infusion although the intra-abdominal pressure didn't exceed 5 em water. This pain was controlled by traditional analgesic. It is worth to mention that no single case of intra-abdominal sepsis or residual collection was recorded after PPL technique. Figure 4

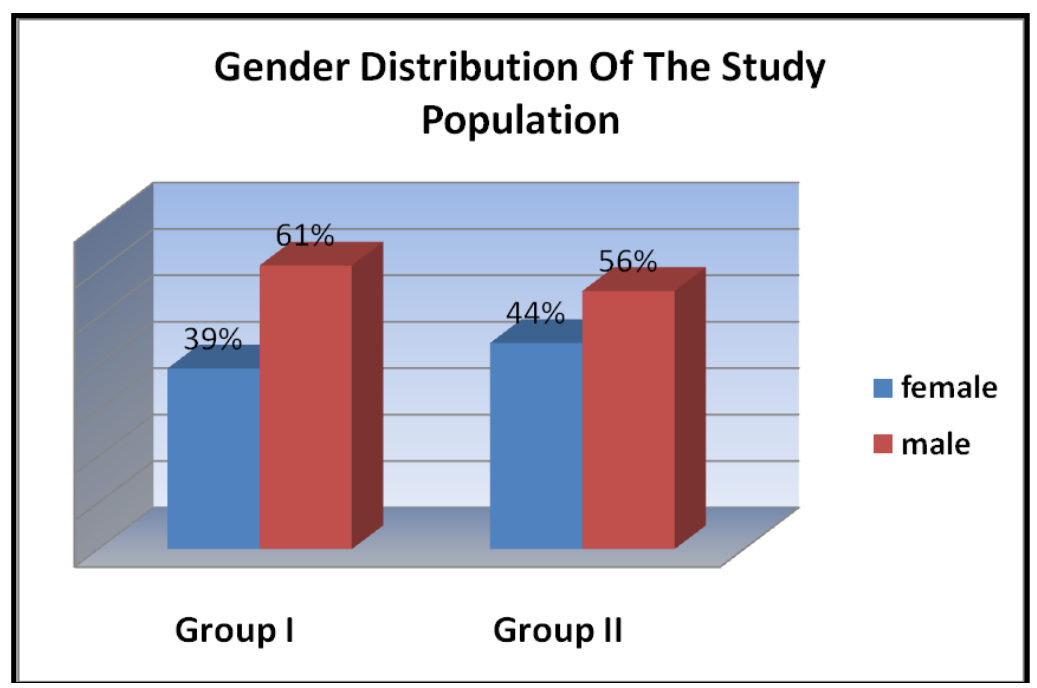

Figure (1): Gender distribution of the study population 
Table (1): Frequency Of Previous Operations in both groups.

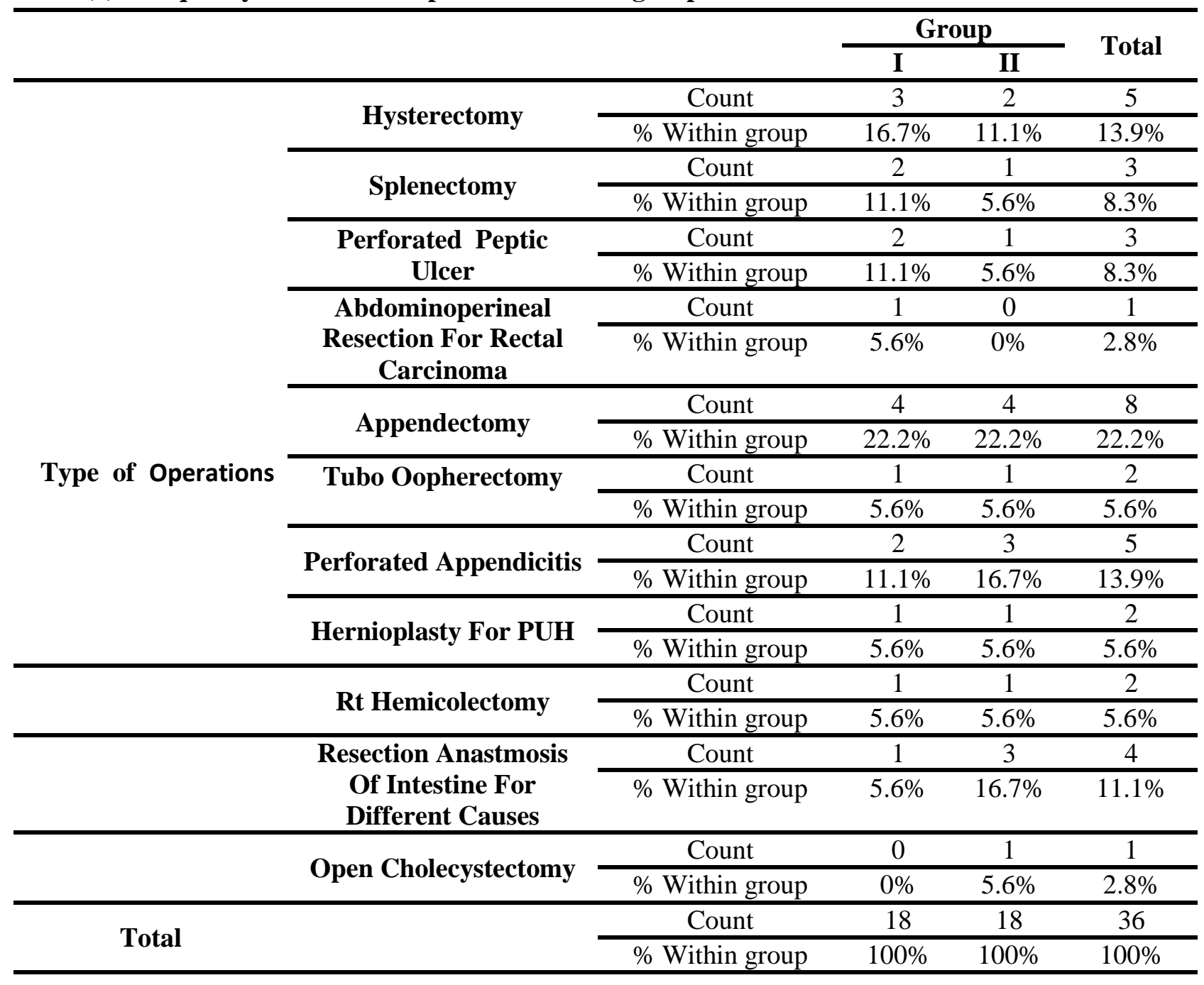

Table (2): Incidence of Recurrence in both groups.

\begin{tabular}{|c|c|c|c|c|c|}
\hline & & & \multicolumn{2}{|c|}{ GROUP } & \multirow{2}{*}{ Total } \\
\hline & & & I & II & \\
\hline \multirow{4}{*}{$\begin{array}{l}\text { Incidence Of } \\
\text { Recurrence }\end{array}$} & \multirow{2}{*}{$\begin{array}{c}\text { NO } \\
\text { RECURRENCE }\end{array}$} & Count & 17 & 11 & 28 \\
\hline & & $\%$ Within group & $94.4 \%$ & $61.1 \%$ & $77.8 \%$ \\
\hline & \multirow[t]{2}{*}{ RECURRENCE } & Count & 1 & 7 & 8 \\
\hline & & $\%$ Within group & $5.6 \%$ & $38.9 \%$ & $22.2 \%$ \\
\hline \multirow{2}{*}{\multicolumn{2}{|c|}{ Total }} & Count & 18 & 18 & 36 \\
\hline & & $\%$ Within group & $100 \%$ & $100 \%$ & $100 \%$ \\
\hline
\end{tabular}




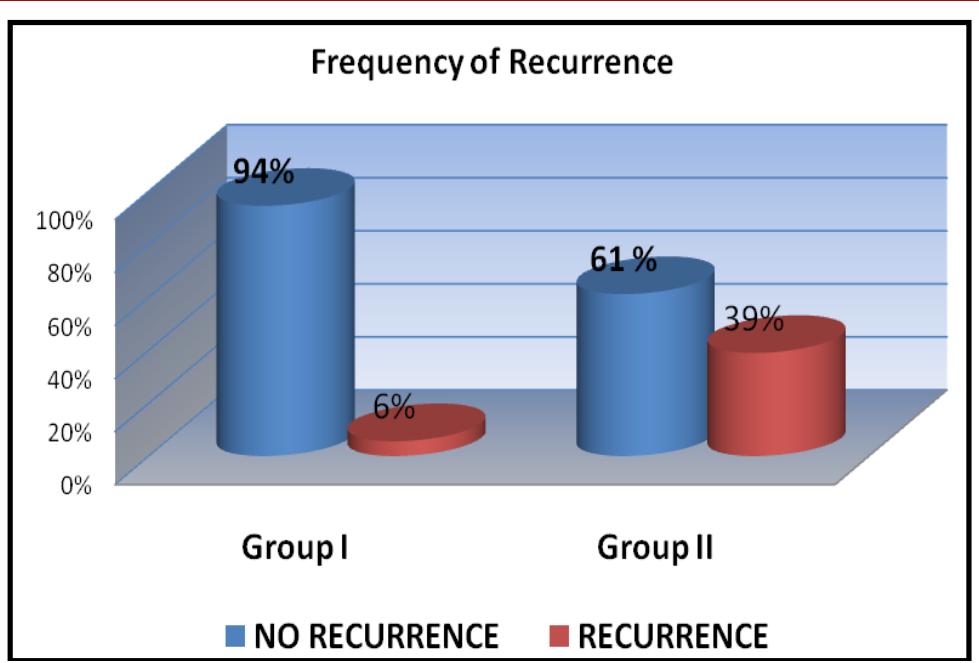

Figure (2): Incidence of Recurrence in both groups.

Table (3): Incidence of REDO in both groups.

\begin{tabular}{|c|c|c|c|c|c|}
\hline & & & \multicolumn{2}{|c|}{ GROUP } & \multirow{2}{*}{ Total } \\
\hline & & & $\mathbf{I}$ & II & \\
\hline \multirow{4}{*}{$\begin{array}{c}\text { Incidence Of } \\
\text { REDO }\end{array}$} & \multirow[t]{2}{*}{ NO REDO } & Count & 18 & 14 & 32 \\
\hline & & \% Within group & $100 \%$ & $77.8 \%$ & $88.9 \%$ \\
\hline & \multirow[t]{2}{*}{ REDO } & Count & 0 & 4 & 4 \\
\hline & & $\%$ Within group & $0 \%$ & $22.2 \%$ & $11.1 \%$ \\
\hline \multirow{2}{*}{\multicolumn{2}{|c|}{ Total }} & Count & 18 & 18 & 36 \\
\hline & & $\%$ Within group & $100 \%$ & $100 \%$ & $100 \%$ \\
\hline
\end{tabular}

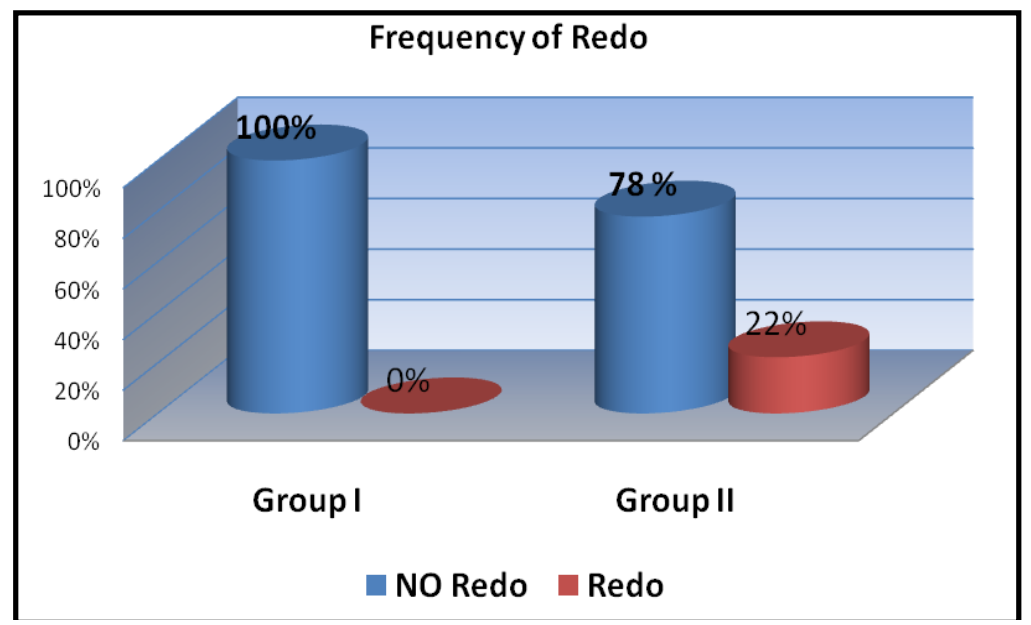

Figure (3): Incidence of redo in both groups. 


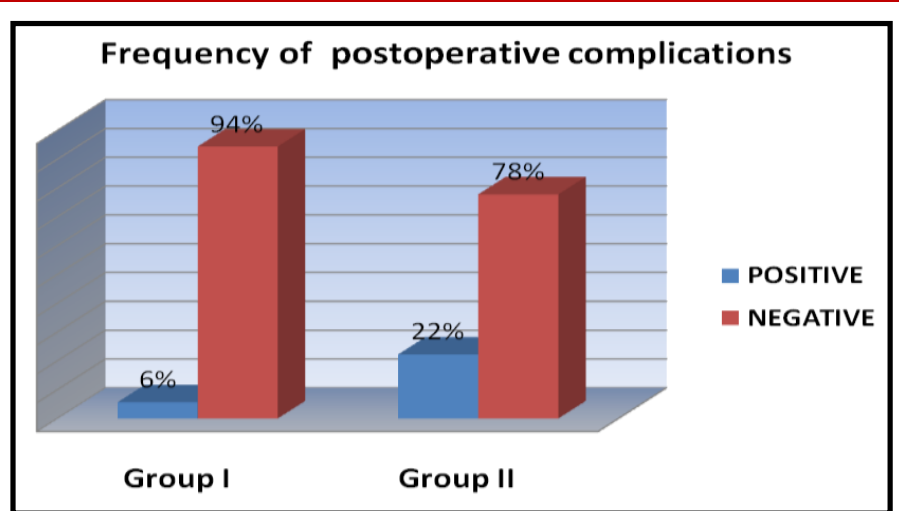

\section{Figure (4): Frequency of postoperative complications in both groups}

\section{DISCUSSION}

The etiology of adhesion formation remains incompletely understood, and, in spite of advances in surgical techniques, there is little change in the epidemiology of adhesions. This is even so in the era of laparoscopic surgery. Adhesions continue to be mysterious not only in terms of their occurrence but also because of their complications which can occur as early as few days after surgery or remain dormant for several years after the initial procedure (14)

In this study only one patient $(5.6 \%)$ in group I developed recurrent ASBO within the follow up period while in group II seven patients (38.9\%) presented with picture of recurrent ASBO. there is significant reduction in the recurrence rate in group $\mathbf{I}$ in comparison to group II.

Among 18 patients of our group I only one case (5.6\%) of recurrent ASBO occurred which respond to conservative measures and needed no surgical intervention i.e that the rate of surgical redo in these patients was zero. in comparison to Fazio et al(4). Who used seprafilm as a barrier to intestinal adhesions who recorded no significant difference between treatment and control group in overall rate of ASBO but the incidence of ASBO requiring re-operation was significantly lower for seprafilm patients. Compared with no-treatment patients

While In Group II, It was found that $\mathbf{1 1}$ patients $(61.1 \%)$ had no recurrent attacks of adhesive intestinal obstruction and 7 patients (38.9\%) had recurrent attacks adhesive intestinal obstruction. 4 patients ( $22 \%$ ) of them had redo operations and $\mathbf{3}$ patients treated conservatively.

The idea of PPL method is that by diluting and washing out of fibrinogen in the peritoneal fluid, we can minimize adhesions among intestinal loops and by creating of some sort of artificial ascitis, fluid acts as a lubricant over the intestinal wall that facilitates motility and prevents adhesions.

The incidence of recurrent ASBO among group II was (38.9\%) which falls in the same range recorded by previous studies which was 19 to $53 \%$ (15).

Also $50 \%$ of this recurrent ASBO occurred in the $1^{\text {st }} 6$ months after surgery, the finding that parallels with data obtained by Fazio et al(4), were they recorded that 50\% of 1 st ASBO episodes occurred within 6 months after the initial surgery (4).

CONCLUSION AND RECOMMENDATION

Postoperative Peritoneal Lavage (PPL) is a new hope for prevention . of recurrent ASBO that achieved significant reduction in recurrence rate and hopefully will abolish the need for surgical re-exploration in victims of ASBO.It is hoped, that this method is studied on a larger scale of patients and centers over a longer period of follow up for re-assessment and possible modifications.

\section{REFERENCES}

1- Amornyotin S (2013): Endoscopy, 2, 382 pages, Publisher: InTech

2- Coleman MG, McLain AD and Moran BJ (2000): Impact of previous surgery on time taken for incision and division of adhesions during laparotomy. Dis Colon Rectum, 43(9):1297-9.

3- Parker MC, Ellis H, Moran BJ Thompson JN et al. (2001): Postoperative adhesions: ten-year follow-up of 12,584 patients undergoing lower abdominal surgery. Dis Colon Rectum; 44 (6): 822-30.

4- Fazio VW, Cohen Z, Fleshman JW, et a!. Reduction of adhesive small bowel obstruction by seprafilm adhesion barrier after intestinal resection Dis colon rectum 2006: 49: 1-11.

5- Liakakos T, Thomakos N, Fine PM, Dervenis C and Young RL (2001): Peritoneal adhesions: etiology, pathophysiology, and clinical 
significance. Recent advances in prevention and management. Dig Surg, 18(4): 260- 73.

6- Attard JP and MacLean AR (2007): Adhesive small bowel obstruction: epidemiology, biology and prevention. Can J Surg; 50 (4): 291: 300.

7- Ivarsson ML, Holdmdahl L, Franzen G, et al. Cost of bowel obstruction resulting from adhesions. Eur J Surg1997; 163:679-84.

8- Ray NF, Denton WG, Thamer M, et al. abdominal adhesiolysis : inpatient care and expenditures in the Unitedstates in 1994. JAm Coll Surg 1998;186;1-9.

9- Cheong YC Laird SM, Li TC, et a!.Peritoneal healing and adhesion formation/ reformation. Hum reprod Update 2001; 7 556-66.

10- Reijnen MM, Bleichrodt RP, Van Goor H. Pathophysiology of intra- abdominal adhesion and abscess formation, and the effect of hyaluronan. Br J surg 2003; 90:533-41.

11- Dijksra FR, Niuewenhuijzen M, Reijnen MM, et al. recent clinical developments Pathophysiology, epidemiology, diagnosis and treatment of intra-abdominal adhesions. Scand J Gastroenterol suppl 2000; 232:52-9.

12- Reed KL, Fruin AB, Bishop- bartolomei $\mathrm{KK}$, et al. Neurokinni-1 receptor and substance $\mathrm{P}$ messenger RNA levels increase during intraabdominal adhesion formation. J Surg 2002; 108:165-72.

13- Ellis H, Moran BJ and Thompson JN (1999): Adhesion-related hospital readmissions after abdominal and pelvic surgery: a retrospective cohort study. Lancet; 353: 1476-80.

14- Al-Salem AH and Oquaish M (2011): Adhesive Intestinal Obstruction in Infants and Children: The Place of Conservative Treatment International Scholarly Research Network. ISRN Surgery, Volume 2011, Article ID 645104, 4 pages.

15- Barkan H, Webster S, Ozeran S. Factors predicting the recurrence of adhesive small bowel obstruction. Am J Surg. 1995:170:361365 . 